\title{
Quasar Variability: New Surveys and New Models
}

\author{
M.R.S. Hawkins \\ University of Edinburgh, Royal Observatory, Blackford Hill, Edinburgh \\ EHg $3 H J$, Scotland, UK
}

\begin{abstract}
In this paper results from a monitoring programme of a large sample of quasars comprising regular yearly observations over a period of 23 years are presented. Structure functions of the light curves are calculated and compared with predictions for models of quasar variability of current interest. These include recently published models of varibility from accretion disk instability, variability from starbursts or supernovae, and variations caused by the microlensing effect of compact bodies along the line of sight. The analysis favours the accretion disk model for low luminosity AGN, but suggests that the variations of more luminous quasars are dominated by microlensing.
\end{abstract}

\section{Introduction}

It has long been understood that one of the keys to understanding the structure of active galactic nuclei (AGN) lies in the nature of the observed variability of their flux. Short term variations were used shortly after the discovery of the first quasars to put limits on the size of the emitting region, but it seems fair to say that since then, optical variability has been hard to characterise and use to constrain AGN structure. In fact much effort has gone into quasar monitoring programmes (Cristiani et al. 1996; Hawkins 1996; Hook et al. 1994) but the results have been hard to interpret. There appear to be several reasons for this. Perhaps the most significant is that quasars vary with most power on a timescale of decades, and monitoring programmes have been in existence this long. A related problem concerns the need for evenly spaced homogeneous data. Few monitoring programmes have achieved this, presenting serious difficulties in analysis.

There are also other difficulties independant of the quality of the data available. To distinguish between various quasar models, predictions of the spectrum of fluctuations must be made which can be compared with the observations. Until recently, little was published in this area, and even general expectations seemed to be at odds with observed timescales and amplitudes of variation. However, a big step forward was made with the publication (Kawaguchi et al. 1998) of predicted structure functions for a model of accretion disc instability, together with similar predictions for a starburst model of variability. In this paper we test these models together with predictions for microlensing against the observed structure function for a large sample of quasars and Seyfert galaxies. 
$\kappa_{*}=0.2$

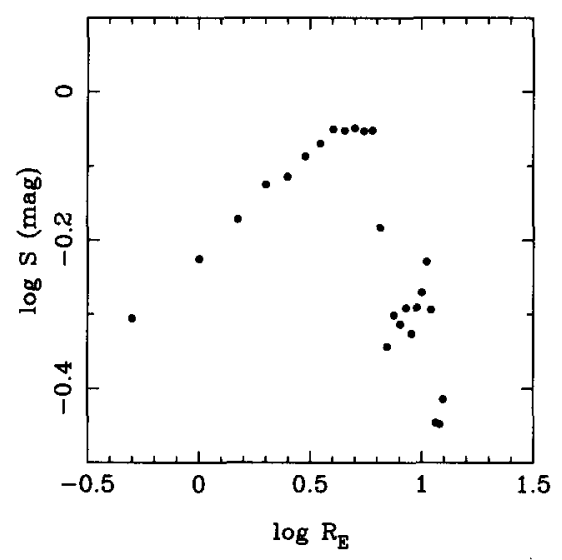

$\kappa_{*}=0.5$

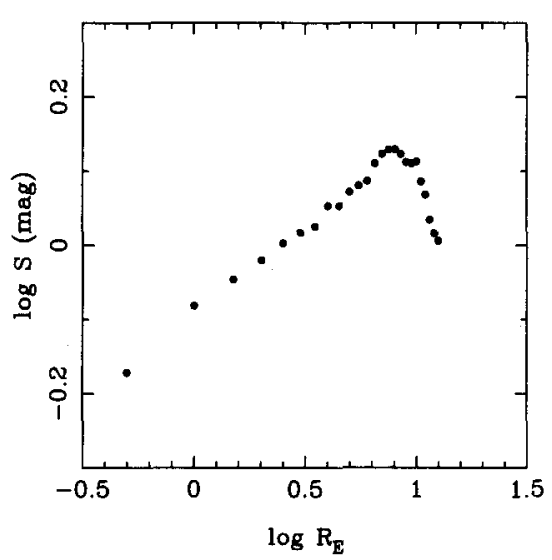

Figure 1. Structure functions for simulated microlensing light curves fo model $b$ of Schneider \& Weiss (1987) (left hand panel), and from Fig. 2(b) of Lewis et al. (1993) (right hand panel).

\section{Model Predictions}

In the standard model of AGN, the central power source is taken to be a massive black hole powering an accretion disc. The most obvious source of the observed flux variations is some form of instability or oscillation in the accretion disc, but so far this has proved very hard to model in such a way that the predicted variations are at all like the observed ones, even for basic parameters such as timescale and amplitude. Furthermore there has so far been little published in the form of quantitative statistical predictions which can be compared with observations.

A great step forward has recently been made by Kawaguchi et al, (1998) with their cellular-automaton model of accretion disk instability. They use this model to generate artificial light curves from which they calculate structure functions which can be compared with those of observed AGN light curves. The overall shape of the model structure functions indicates a power law relationship with inreasing power on long timescales, until an eventual turnover. Although the amplitude of the variations and the timescale of the break are dependent on the free parameters in their model, the logarithmic slope of the structure function is robust, with a value of $0.44 \pm 0.03$.

Kawaguchi et al. (1998) also carry out a similar analysis for the starburst model of AGN variability (Aretxaga \& Terlevich 1994). In this model the nuclear light of AGN is the result of a sequence of supernova explosions, and the observed variations come from the stochastic nature of the burst timings. Kawaguchi et al. (1998) produce simulated light curves based on this model, and as for the accretion disc model, calculate structure functions. Again, the overall shape shows a power law relationship with a long timescale turnover, the position of 
which is dependent on model parameters. However, as with the accretion disc model, the logarithmic slope of the structure function is relatively stable with a value of $0.83 \pm 0.08$.

As well as models for the intrinsic variability of AGN, it has also been argued (Hawkins 1993) that the observed variations in quasars may be dominated by the effects of gravitational microlensing. In this picture it is postulated that a population of compact bodies criss-cross the line of sight to the quasar, causing the light to fluctuate from the effects of gravitational lensing. A number of groups have carried out simulations of the resulting light curves (Lewis et al. 1993; Schneider \& Weiss 1987), and they have been published in such a form that it is possible to calculate the structure functions for comparison with the data.

Fig. 1 shows structure functions for long runs of simulated data from two different groups, digitised to enable comparison with observations. The data is presented as a function of $R_{E}$, the Einstein radius of the lenses, and to turn this into a timescale an average velocity across the line of sight must be applied. The relation has a power law form with a break towards long timescales. The power is dependent on the optical depth of the lenses but the logarithmic slope appears to be robust for different simulations with a value of $0.25 \pm 0.03$.

It will be seen that the three models we have considered in this section all predict structure functions with a power law form increasing in power towards an eventual turnover at long timescales. However, each model makes a different prediction for the logarithmic slope of the structure function, that is, $0.83 \pm 0.08$, $0.44 \pm 0.03$ and $0.25 \pm 0.03$ for the starburst, disc instability and microlensing models respectively. All three slopes are significantly separated from each other, and are well suited for comparison with observations to determine the most favoured model of variability.

\section{Observed Structure Functions}

Over the last 25 years or so, a large scale quasar minitoring programme has been undertaken using automated measures of UK $1.2 \mathrm{~m}$ Schmidt plates (Hawkins 1996) taken on a variety of timescales from hours to years. Of particular interest for the present work is an unbroken series of yearly observations from 1977 to 2000 in the $B_{J}$ passband (IIIa-J/GG395 emulsion/filter combination). The plates have a useful limiting magnitude of $B_{J} \sim 21.5$ and the monitoring area comprises the central $19 \mathrm{deg}^{2}$ of the Schmidt field. Some 180,000 objects were detected in this area of which around 1500 are likely to be quasars. So far 610 of these have been confirmed as quasars with measured redshifts. Each of these has a light curve covering 23 years and together they form the parent sample for the present investigation.

Initial examination of the light curves from the monitoring programme suggested that the low luminosity AGN with $M_{B}>-23$ (Seyfert galaxies) varied in a qualitatively different way to the more luminous objects with $M_{B}<-23$ (quasars). Accordingly, in the first instance the observed structure function was calculated for quasars alone. The result is plotted in Fig. 2, and it will be seen that it has an approximately power law form, with a logarithmic slope of $0.20 \pm 0.01$. 


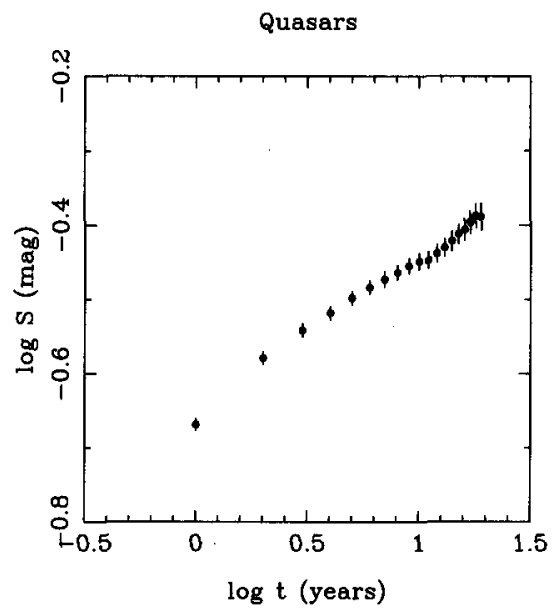

Figure 2. Structure functions for the light curves of a sample of 401 quasars from the survey of Hawkins (1996).

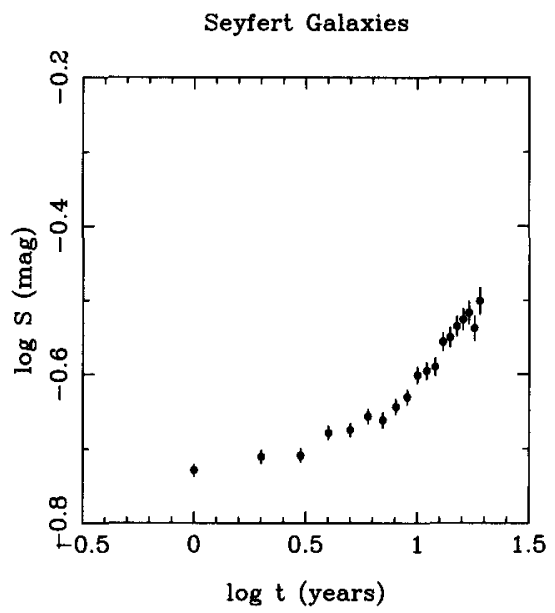

Figure 3. Structure functions for the light curves of a sample of 45 Seyfert galaxies from the survey of Hawkins (1996). 
NGC 5548

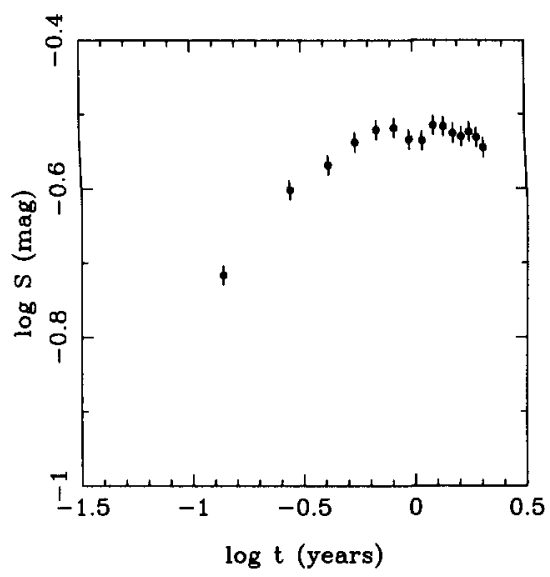

NGC 5548

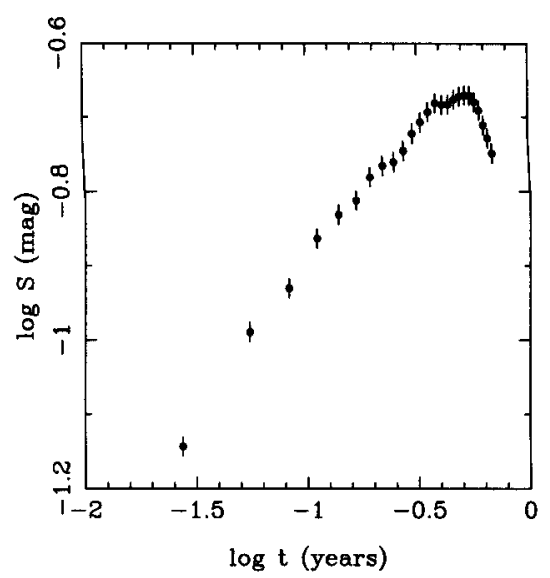

Figure 4. Structure functions for the light curve of NGC 5548 from Peterson et al. (1999) and references therein for 50 day intervals (left panel) and 10 day intervals (right panel).

If we now turn our attention to the Seyfert galaxies in the sample a rather different picture emerges. The structure function for these low luminosity objects is shown in Fig. 3 and it is immediately clear that there is much less power than for the quasars. Of more importance for the present investigation is the fact that the slope of the linear part is $0.36 \pm 0.02$, significantly steeper than for quasars. Towards short timescales the structure function becomes flat, which appears to be where the signal becomes dominated by noise.

This result can be tested by making use of results from the extensive monitoring programme of NGC 5548 by Peterson et al. (1999). This Seyfert galaxy has been monitored for many years on a timescale of a few days in optical wavelengths, and Fig. 4 shows the result of calculating structure functions from parts of the data sampled in a uniform manner. The left hand panel sampled at 50 day intervals shows a structure function with a long timescale turnover, which never really becomes truly linear at shorter timescales. In the right hand panel the data is sampled every 10 days, and here it is clear that there is an extended linear portion, with logarithmic slope of $0.38 \pm 0.01$. This is close to the value obtained for the Seyfert galaxies in the wide field monitoring programme.

\section{Discussion}

The results of the preceding two sections are summarised in Table 1. For Seyfert galaxies it is clear that the favoured model for the observed variations is accretion disc instability. The starburst model is ruled out at a high level of significance, and there appears to be no reason to attribute the variations to microlensing. 
Table 1. Structure Function Slopes

Model Predictions

$\begin{array}{ll}\text { Model } & \text { Slope } \\ \text { Starburst } & 0.83 \pm 0.08 \\ \text { Disc Instability } & 0.44 \pm 0.03 \\ \text { Microlensing } & 0.25 \pm 0.03\end{array}$

Observations

$\begin{array}{ll}\text { AGN Class } & \text { Slope } \\ \text { Seyfert Galaxies } & 0.36 \pm 0.02 \\ \text { Quasars } & 0.20 \pm 0.01\end{array}$

For quasars, the position is different. Here again the starburst model is ruled out, even more strongly, but the accretion disc instability model is also inconsistent with the observations. It is of course possible that the parameter space explored by Kawaguchi et al. (1998) is too small, or that other disc instability models might make different predictions for the structure function slope; future work may well clarify this. For the moment, the best match for the observed quasar structure function is from the predictions for microlensing.

The implication of Table 1 is that, while Seyfert galaxy variation is caused by accretion disc instability, quasar variability is dominated by the effects of microlensing. The most plausible explanation for this would appear to be that at low luminosity the observed variations in AGN are caused by accretion disc instability, but for more luminous objects intrinsic variations become smaller in amplitude. However, at the higher redshifts at which quasars are found, microlensing becomes dominant due to the greater optical depth of lenses.

\section{References}

Aretxaga, I. \& Terlevich, R. 1994, MNRAS, 269, 462

Cristiani, S., Trentini, S., La Franca, F., Aretxaga, I., Andreani, P., Vio, R., Gemmo, A. 1996, A\&A, 306, 395

Hawkins, M.R.S. 1993, Nature, 366, 242

Hawkins, M.R.S. 1996, MNRAS, 278, 787

Hook, I.M., McMahon, R.G., Boyle, B.G., Irwin, M.J. 1994, MNRAS, 268, 305

Kawaguchi, T., Mineshige, S., Umemura, M., Turner, E.L. 1998, ApJ, 504, 671

Lewis, G.F., Miralda-Escudé, J., Richardson, D.C., Wambsganss, J. 1993, MN-

RAS, 281, 647

Peterson, B.M. et al. 1999, ApJ, 510, 659

Schneider, P., Weiss, A. 1987, A\&A, 171, 49 